\title{
Nonhuman primate neuroimaging opens up
}

\author{
To bridge brains and make the most of their nonhuman primate models, neuroscientists are setting standards, \\ building tools, and starting to share their neuroimaging data.
}

\author{
Ellen P. Neff
}

S ituated in each of our frontal lobes is a small, elongated bit of tissue called Area 55b. It's one of 180 recently delineated cortical areas per hemisphere, identified with magnetic resonance imaging (MRI) ${ }^{1}$.

But not all Area 55b's are alike. Imaging data recorded from over 200 human subjects reflected our individuality. In some people, Area $55 \mathrm{~b}$ is a single elongated area in the frontal lobe; in others, it's split into two parts with a neighboring area intervening. "The idea that we all have the same basic organization still remains robust, but if you look at it in detail, our brains are not just like one planet... we have billions of 'planets' and each one is a slight variation of the other," says David Van Essen, a neuroscientist at Washington University in St. Louis who has long studied both the human and the nonhuman primate brain. "The more we can understand those differences in cortical functional organization, the better we can hope to understand what makes each of us a unique individual."

Van Essen refers to himself as a 'cortical cartographer.' Though technology has progressed substantially since his early days with pencils and tracing paper, creating maps of the cerebral cortex remains a convoluted process. Likening brains to celestial bodies, he and his lab take a surface-based approach. "We have to make representations to smooth out the wrinkles in much the same way that maps of the Earth's surface smooth out the bumps of mountains and valleys to make flat, two-dimensional representations," he says.

Such a geographical approach can streamline the problems neuroscientists run into when trying to compare and contrast brains-of humans, and of the nonhuman primates studied as models of various aspects of human health and disease. With complex behaviors and cognition and a number of similarities to the human brain, nonhuman primates are especially valuable in neuroscience research. Increasingly sophisticated nonhuman primate models of specific neurological disorders can be made with transgenic and other genome engineering methods, and with advancing technologies the animals' brains can be

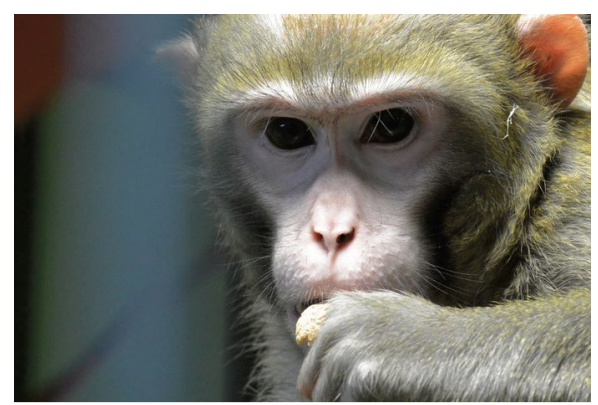

Alvar, a rhesus macaque at Newcastle University. Credit: Petkov lab

explored from across the entire organ down to individual neurons.

Our brains though are fundamentally different from other primates, says Van Essen. "It's like comparing a map of the Earth with a map of Mars," he says. "How do you align two maps that have evolved in such a way that some regions are very similar across species, and some are very different?"

It's a question many neuroscientists need to tackle to make the most translational use of their nonhuman primates. Finding the fundamentals that are shared both between individuals and between species provides a measure to compare against when researchers are trying to identify and treat diseases that deviate from that norm.

"The information that we can obtain in nonhuman primates, in ways that you can't in humans, can nonetheless be directly linked up to certain disorders that we on work with our clinical partners," says Christopher Petkov, a neuroscientist at Newcastle University. "You need an approach in which you can translate that information to humans. Neuroimaging, which you can do in both, ends up being an important bridge."

But work in nonhuman primates has often been a lab-by-lab endeavor that tries for ethical reasons to use as few animals as possible. "If you're recording neurons or doing brain imaging, typically it's going to be: one is a case study, two is a population," says Petkov. "And folks are getting data in very different ways. Even if they're happy to share by way of publication, it's just not practical to combine those data sets and do anything more with them."

The time is coming to team up, and to open up. "The complexity of the brain-the human brain, the monkey brain -is so much greater than we had realized even a few years ago. It outstrips what one lab can acquire and analyze and interpret on their own," says Van Essen. A leader of the Human Connectome Project, an initiative launched in 2009 to map the human brain, Van Essen has long been an advocate for data sharing. What started as an uphill battle in the late 90s and early 2000s has nevertheless been progressing. "The transition has been fairly dramatic just in the past few years," he says.

Sharing is bearing fruit for human neuroscience research. "The human imaging community wanted to start sharing data in a very systematic way, and analyzing that data, preprocessing it-just to make it easier for people to access that data," says Petkov. "And all of a sudden, the scientific community has access to thousands of data sets-it's proven extremely useful for scientific discovery."

Now, many in the nonhuman primate community hope to follow suite with their own collaborations to standardize procedures, start sharing their MRI data, and continue building that bridge between neuroscience in monkeys and neuroscience in humans.
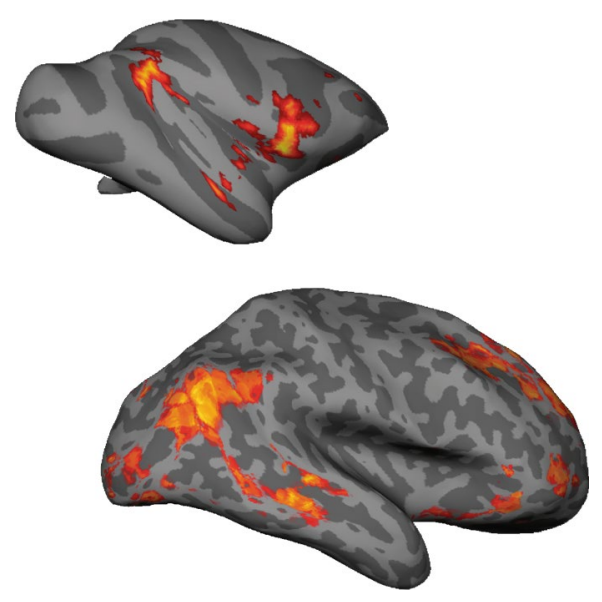

Macaque vs. man. Credit: Petkov lab 


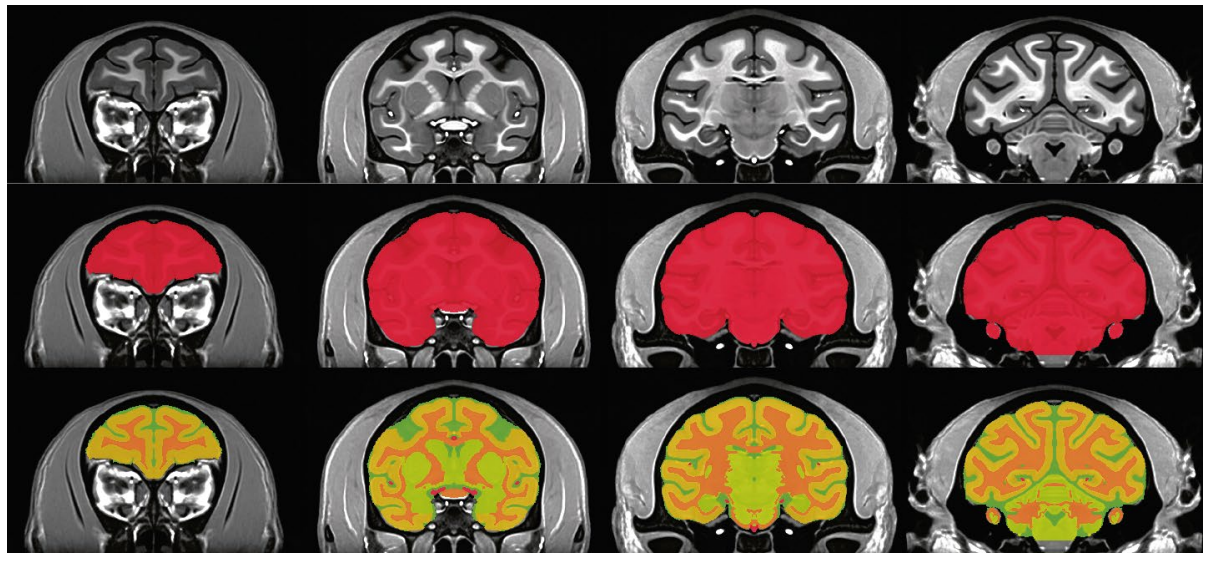

A montage of (top) coronal slices through the NIH Macaque Template, (middle) a mask of the brain, and (bottom) the segmentation of 5 main tissue types-gray matter, deep gray matter, white matter, cerebrospinal fluid, and blood vessels. Image reprinted with permission from Seidlitz et al. ${ }^{2}$,

(Elsevier, Ltd.) 2018

\section{To templates}

Since its advent in the 1980s, MRI has become widespread in both clinical and preclinical applications. As a non-invasive neuroimaging modality, MRI enables animals to be imaged repeatedly and an entire image of the brain to be built, and then traversed, in 3D. "This ability of MRI is virtually unparalleled by any other imaging modality," says Afonso Silva of the University of Pittsburgh.

In the ensuing decades, the human field has converged around a handful of standard protocols for data acquisition, processing, and analysis. The canonical human neuroimaging templates, for example, allow translation between studies based on defined coordinates in the brain, upon which increasingly sophisticated bioinformatics tools can be set to work.

What's become common for human primates remains less so among their nonhuman counterparts.

Though specialized large-animal MRI machines exist, they tend to be expensive and few and far between. Most macaque labs instead rely on equipment and tools that have been optimized for use in humans. A big issue is with the bore-the opening into magnet of the machine. The smaller the bore relative to the subject inside, the higher the magnetic field and the higher image resolution \& quality possible, says Silva. A machine built to fit an adult human leaves empty space around smaller nonhuman primates, such as rhesus macaques. Labs need to take that into account, and as a result many adjust for it differently in their imaging and analysis protocols.

That quickly proved to be a hurdle to a cross-species comparison Jakob Seidlitz had planned while working at the National
Institutes of Health (NIH). Compared to human subjects, there was no standard way to acquire, process, or analyze structural and functional neuroimaging data from nonhuman primates. "I really needed an automated way to extract multiple morphometric features from the cortex in the monkeys, and there was no automated way to do this," says Seidlitz. "The human pipelines, as applied to macaques, were not sufficient."

Given that most macaques at the NIH are imaged with the same handful of machines, Seidlitz saw an opportunity to help create a resource for that community, "to bridge the gaps that differ between experimenters and try to standardize at least how we're analyzing the structural data," he says. The hardest part? Getting buy in.

"A lot of this data, it's very precious," he says. To facilitate, they asked for structural data that people weren't looking at directly. "Most people collect structural data as a way to register other types of data, such as functional neuroimaging data," he says. In the end, Seidlitz and his colleagues aggregated structural MRI imaging from 31 macaques ( 25 males and 6 females) at the NIH colony, which they combined into a new high-resolution macaque brain template, the National Institute of Mental Health Macaque Template (NMT), as well as associated pipelines for processing structural data ${ }^{2}$.

But there is a bigger primate research community that might benefit from such a template, and much more imaging data that can, with the right tools, be combined to make larger, richer datasets for neuroscientists to explore.

Michael Milham, a cognitive neuroscientist at the Child Mind Institute in New York,

\section{Getting primed}

was used to working with big numbers. In human neuroimaging, a particular study can include thousands of subjects. As he shifted to nonhuman primate work to validate what he was finding in child and adolescent patients and pursue translational work for novel neurotherapies, such as brain electrostimulation, the numbers available to him shrank drastically to single digits. "With small samples, you can't appreciate individual variation, and you really have a wide open question about reproducibility," he says.

With prior experience organizing human data sharing efforts, he's been pushing for similar collaboration in the primate field. In 2018, he and over 60 coauthors presented PRIMatE Data Exchange (PRIME-DE), an "open resource to aggregate anatomical, functional, and diffusion MRI datasets." The first data released include 25 collections from 22 international facilities, encompassing neuroimaging data from 216 primates.

The goal, says Milham, "is to help refine the model, to decrease unnecessary redundancy and collection, and also to ensure the best techniques are being used and that we get the maximum output. Open data sharing absolutely increases the return." PRIME-DE is an opportunity for the community to come together across borders says Petkov, to talk about the kind of data and analytics the field wants, and to solve problems together that each lab would otherwise be solving in their own particular way.

"The more we can share, presumably there will be experiments that may not have to be done," says University of California Los Angeles neuroscientist Michele Basso. Researchers could re-use existing data, rather than having to do an experiment themselves, she says. Sharing could also open animal data to those for whom it is currently out of reach. Take a hypothetical scientist with no primate facility at her university. "Here now is a repository of data that she could go and mine, and she could ask her novel, insightful question that she couldn't otherwise ask, "says Basso." It's that simple."

Last September, an expanded PRIME-DE working group met in London to start

Table 1 | Issues identified by the PRIME-DE working group
1. Standardizing data collection
2. Animal welfare, regulations, and intellectual property
3. Data standards, quality assessment, and analytic software
4. Coordinate paradigm design 


\section{Box 1 | Credit where credit is due}

As data becomes more openly available, people may be looking at the data who haven't directly worked with the animals, notes Seidlitz, currently a post-doc at Children's Hospital of Philadelphia and the University of Pennsylvania. "One big consideration is how to make this inclusive for those people," he says. "[In the future] I don't know that I'm going to be directly working with nonhuman primates, but the questions that I'm interested in would rely on that data. So staying involved and current with that area of research is very critical."

But as he found while building the NMT macaque template, those actually generating the data need an incentive to share it-especially if that data has not yet featured in published research. The PRIME-DE participants believe they have a solution in what they call the Collaboration Model: data generators can advertise what they've collected, collaborators with relevant expertise can get involved, and all involved will get credit at publication-at which time the data itself will be made public as well.

That seemed reassuring to many notes Petkov, but "[the institutions] need to step up and recognize that they need to base their promotions on data sharing and some of these initiatives, and encourage their folks to be able to do that." and describing MRI datasets developed by researchers at Stanford ${ }^{5}$. Quality among the primate datasets will likely vary from data set to data set for the time being, necessitating documentation about species-specific details, protocols, and data processing steps in the metadata that accompany the files. But, it should be worth the effort.

"If you structure data like that, you can pull any big pipeline and just apply it to your data," says Katja Heuer, a PhD student at the Max Planck Institute for Human Cognitive and Brain Sciences working with Roberto Toro's lab at Institut Pasteur in Paris. "It will work." Such has been the case of late in the human realm, where there are ample datasets in standard formats, for which a number of analysis tools have been developed. The less common the species working through the details that aren't quite so simple: dealing with all those different ways people work with and image their animals. They identified four main areas to address (Table 1$)^{4}$, with discussions surrounding data acquisition and standards, crediting (Box 1), licensing and regulations, and analysis tools and protocols.

While consensus on truly universal protocols and standards may take time, the participants identified a number of areas where more information can and should be documented to help different labs interpret what another has done. For example, the regulations under which the animal studies are conducted, how awake animals are trained and/or immobilized in the machines, or anesthesia protocols used should be shared so that others can take those details into account when comparing or combining data.

Regarding anesthesia, there actually seemed to be a high degree of commonality, says Milham, "which was reassuring. On the flip side, if you asked people how they analyze their data, everyone was talking about tweaks. And the problem is that the nonhuman literature is forced to use tools that were developed for human imaging, so they all tweak it and that comes with a lot of issues with signal variations and data quality." There, the field is still sharing ideas for how best to move foward.

\section{Macaques and more}

Getting different neuroimagng data into a standard format will be necessary for quality control and interoperable sharing. The PRIME-DE data releases take advantage of the Brain Imaging Data Structure (BIDS) format, a data standard for organizing

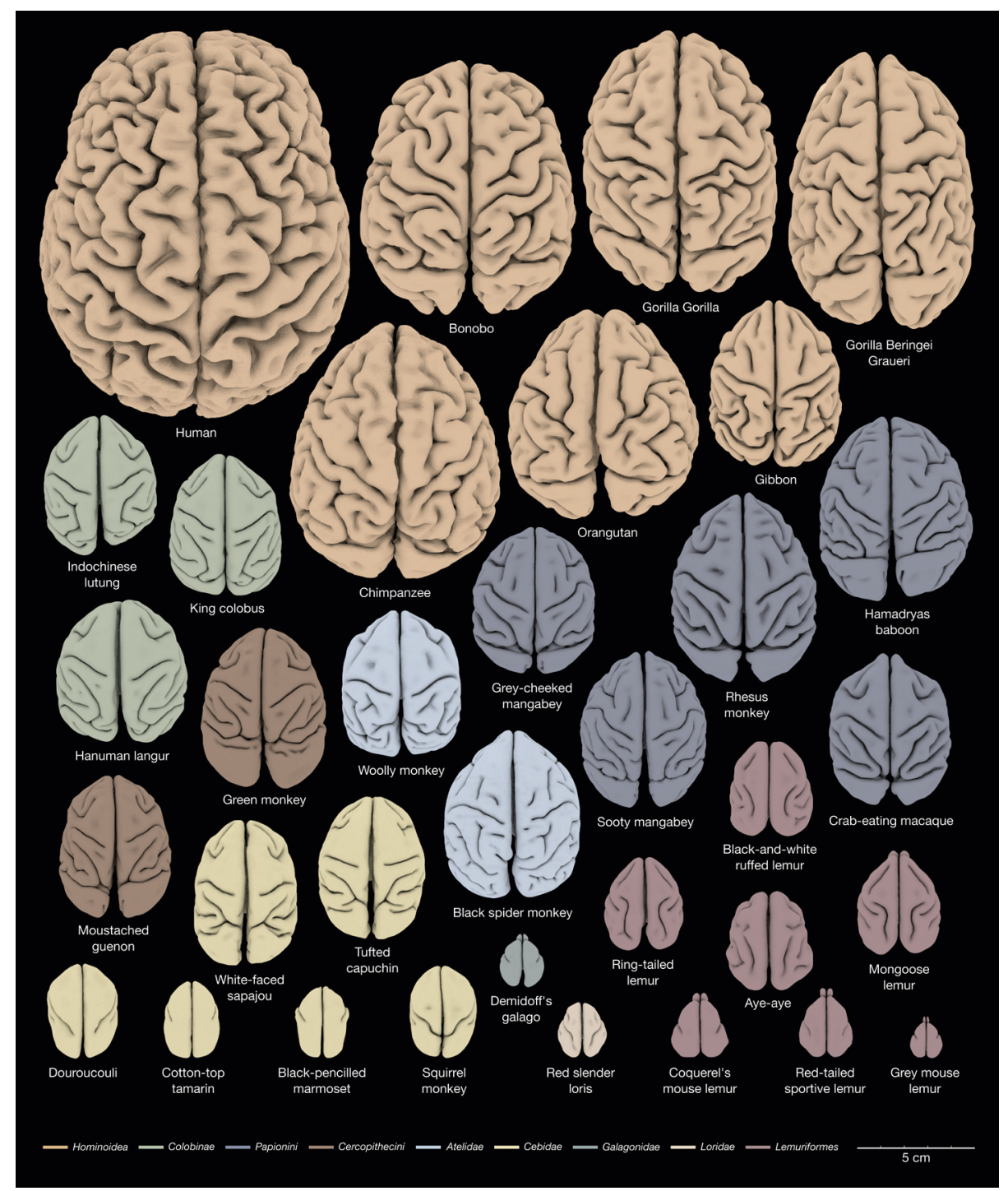

All across the primate tree-dorsal view of the brains of 34 different species, from human to grey mouse lemur. Credit: Katja Heuer \& Roberto Toro, from https://doi.org/10.1101/379750 


\section{Box 2 | MRI \& Marmosets}

Silva has spent much of his career working with MRI images, but he came to the nonhuman primate world not by way of humans, but laboratory rodents. And his primate is not a macaque, but a marmoset. Although marmoset brains are lissencephalic - smooth, rather than folded like ours or a macaques-they nevertheless share many of the same anatomical and functional areas of other primates. The marmosets come with a notable advantage for neuroimaging: their small size means they can fit in small animal MRI machines. "If the marmosets were larger, we'd be in the same boat as macaques, and we would have to use the human machines," he says.

That snug fit permits much higher resolution images to be recorded than is currently possible, in macaques or in humans. In his latest publication using a post-mortem marmoset brain, he and his team were able to visualize and map (lacking established references, albeit by hand) the white matter connections that streak the animal's brain ${ }^{8}$. That white matter data joins Silva's first 3D MRI-based atlas, of the marmoset cortex, and will soon be followed by a population atlas looking across different individuals. As more neurological models are developed, that will help marmoset researchers understand the norm and deviations from it, says Silva.

Beyond MRI, Silva is also applying other imaging methods to his marmosets. Another non-invasive approach is position emission tomography (PET) which tracks ligands that bind to specific proteins, such as the Presenilin 1 protein often found in patients with familial Alzheimer's disease. A marmoset model, currently being developed, could then be tracked over its entire life to look for early signs of triggers of disease onset. however, the less likely for there to be such resources. Progress is being made for macaques, says Heuer, "but outside of this species, we're largely on our own."

Heuer is interested in comparative neuroanatomy as a way to understand brain organization in general as well as the sources of normal and pathological variation. That means looking across many different species to find fundamental principles. "We cannot understand everything just by looking at humans," she says. Her first comparison involved 34 different primate species ${ }^{6}$; she and her colleagues are now expanding to study the brains of 150 vertebrate animals, as part of an effort to digitize the Vertebrate Brain Collection of the National Natural History Museum of Paris. In this case the brains are of museum specimens that can undergo long scans in MRI machines to produce high-resolution images.

For many of those animals, it will have been the first time their brains have been imaged; segmenting the brains is thus mostly done by hand, but with the help of a number of analytical tools Heuer and her colleagues have been building (mostly on nights and weekends, she notes), such as the BrainBox and Brain Catalogue. "The web applications that we develop not only facilitate access to that data-they add a layer of collaboration on top," she says.

Users-neuroscientists and citizen scientists alike-can do things like view a particular brain, segment it, and build 3-dimensional surfaces. "We're trying to reach out to anybody who could be interested in the brain, gathering a community, and making data accessible in an intuitive way,' she says. "It's much better to have something than to have nothing, and if an expert isn't happy with a result, they can modify it."

Heuer has been starting to work with machine learning and data scientists to pre-process and automate some of the analysis they are currently doing by hand. Facebook's 3D shape recognition algorithm, for example, works nicely, but it requires thousands of images. "Our collections of vertebrate brain data are much smaller," says Heuer, "But brain shapes tend to follow a common pattern, which we hope deep learning algorithms will be able to capture."

Most of the subjects that contributed to the PRIME-DE datasets so far have been macaques, but the collaboration hopes to expand to other species, including baboons, marmosets (Box 2), and perhaps someday other nonhuman animal species as well. "We don't have to hit every species, but we need to be able to make reasonable jumps which will enable translation," says Milham. "Evolutionary perspectives are essential to understand the origins of individual variation in humans and disease states."

\section{Data shared-now what?}

Though the collection is still growing and data from the full number of animals available has yet to be taken advantage of, the PRIME-DE resource is already starting to bear fruit.

Milham and colleagues recently compared data from three macaque datasets that contained both awake and anesthetized animals. That brought the number of animal subjects up to 48 , and let the team make some comparisons between individuals-a common practice in human research. They noted individual heterogeneity but a conservation of patterns that reflects observations in people ${ }^{7}$.

There are though other scales at play, able to be visualized in animal models in ways not possible in humans. There's the macroscale that MRI capturescentimeter-scale regions of connectivity and functionality across the whole brain; there's a mesoscale, down to micrometer fields; and a microscale-the nanometer size of individual neurons \& axons. "Nonhuman primate work, and nonhuman work in general, provides a unique opportunity to actually link the scales," says Milham.

To reach those smaller scales, more invasive approaches are needed. One example is optical imaging, in which genetically modified animals express calcium indicators that fluoresce as neurons fire. Through a cranial window, "you can directly visualize neuroactivity at the level of individual cells," says Silva. "You can really identify individual cells and individual blood vessels, at the expense of only being able to see a very small area of the cortex at a time."

"We use electrophysiological techniques," says Basso, manipulating neurons via chemical and/or electrical means to try to understand the relationship between a particular area of the brain and a cognitive behavior of interest. Imaging techniques such as 2-photon imaging and microendoscopy methods that have been common in mice are now being translated to nonhuman primates. "The resolution is down to the level of single neurons," says Basso, "You see live cells and the direct activity of those cells."

As data continue to be collected at each of those different scales, connections need to be made. "You have some scientists who are expert at these cellular resolution levels, you have other scientists who are expert at these large scale, network dynamic levels, and the next step is to start bringing these groups together and trying to get them to speak the same language," says Basso. "Statisticians, computational modelers, theoreticiansthese are the people who are going to bring the linkages between these two types of scientists."

Tools will be built to connect different scales, with a goal to view different regions 


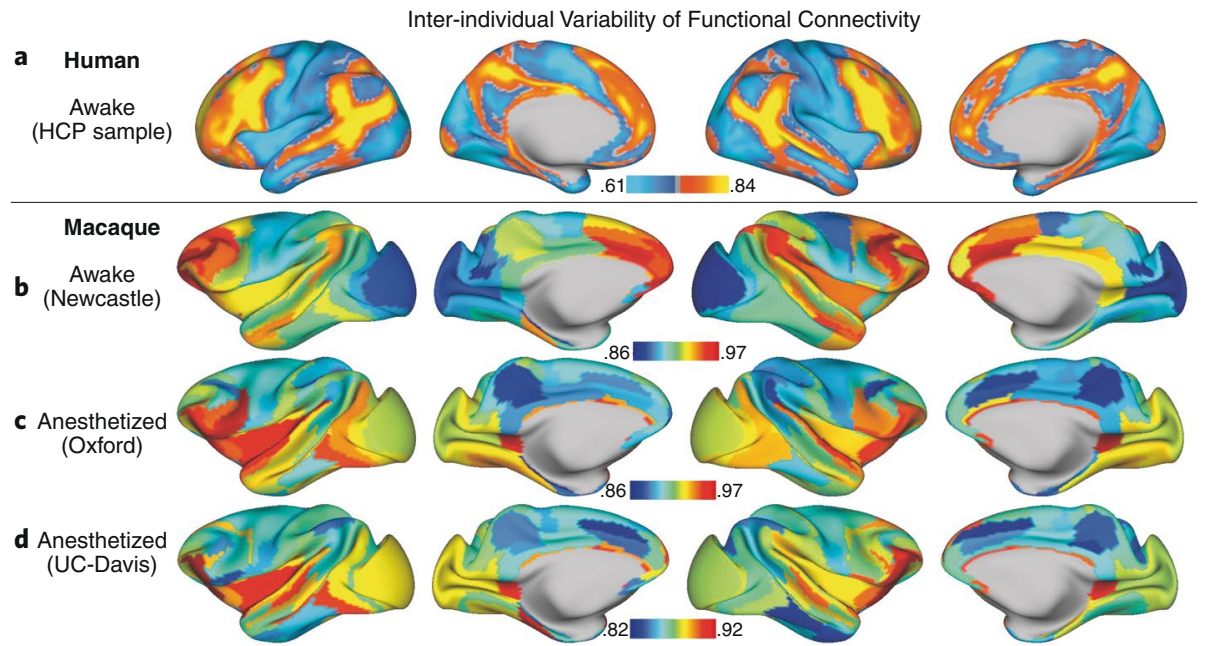

Combining data to study interindividual variation in human, awake macaques, and anesthetized macaques. Image reprinted with permission from Xu et al. ${ }^{7}$ (Elsevier, Ltd) 2019.

of the brain during different circumstances or tasks, drill down to the circuitry involved, and then compare that part of the human brain to different animal models-macaques, rodents, fruit flies, etc. "That's the vision for the next decade or two," says Van Essen. "I like to dream, and I have confidence that the next generation of neuroscientists and neuroimagers and neuroinformaticians will bring it into play."

Doing so will require training across disciplines; some will specialize in the neurobiology — the anatomy, chemistry, and molecular biology of a particular species-and others, the informatics and technology needed. "I think there's going to be a transition towards progressively more team-oriented science, where people know enough to know who they need to collaborate with," says Van Essen.

Open sharing can bring in a wider range of people with different analytical skills, whether or not they work with nonhuman primates. "They will jump in and they will bring their skill sets to bear, and you will see a much more rapid maturation of the analytic capabilities," says Milham. "A much larger workforce essentially emerges with open sharing."

It's still in early days, and community convergence on a number of issues still remain to be sorted out. But the PRIME-DE members are forming working groups and hope to continue to bring in more researchers. "It's becoming a much tighter network," says Seidlitz. "I think in a practical sense, that's what's going to move the field forward in the long run in terms of the science-it opens up new possibilities for asking questions about nonhuman primate brains."

Petkov is encouraged by the reception so far. "Having that community means that folks can get creative about the questions that they ask," he says. "You can expect quite a lot of discovery."

\section{Ellen P. Neff $₫$ \\ Lab Animal.

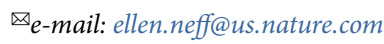

Published online: 24 April 2020

https://doi.org/10.1038/s41684-020-0531-3

References

1. Glasser, M. F. et al. Nature 536, 171-178 (2016).

2. Seidlitz, J. et al. Neuroimage 170, 121-131 (2018).

3. Milham, M. P. et al. Neuron 100, 61-74.e2 (2018).

4. The PRIMatE Data Exchange (PRIME-DE) Global Collaboration

Workshop and Consortium. Neuron 105, 600-603 (2020).

5. Gorgolewski, K. J. et al. Sci Data 3, 160044 (2016).

6. Heuer, K. et al. Cortex 118, 275-291 (2019).

7. Xu, T. et al. Biol Psychiatry Cogn Neurosci Neuroimaging 4, 543-553 (2019).

8. Liu, C. et al. Nat Neurosci 23, 271-280 (2020). 